\title{
Application Effect and Nursing of Non-Invasive Ventilation in Acute Exacerbation of Chronic Obstruction Pulmonary Disease Patients
}

\author{
Zongying $\mathbf{L i}^{*}$ \\ Affiliated Hospital of Xuzhou Medical College, Xuzhou, Jiangsu 221003, China
}

\begin{abstract}
Objective: To study the application methods, summarize the nursing experience and discuss the clinical effect of non-invasive ventilation in acute exacerbation of chronic obstructive pulmonary disease (COPD). Methods: 152 cases of AECOPD patients, who received treatment in our hospital from January 2012 to December 2014, were selected and divided into the experimental group and control group randomly. Patients in control group received conventional treatment, whereas besides conventional treatment, experimental group received non-invasive positive pressure ventilation and relevant nursing management. The changes in arterial blood gas and clinical indicators before and after treatment for both groups were observed. Results: After 7 days treatment, all the results of blood gas analysis were significantly improved $(p<0.05)$. For the respiratory status (based on Borg scale), two groups were significantly improved after treatment, however, experimental group shown better than control group $(p<0.05)$. The two groups had statistically significant difference $(p<0.05)$ in the aspects of prognosis, endotracheal intubation and hospitalization time. As for the death index, there was no significant difference between two groups. No special treatment was needed for adverse reactions, they would be relieved slowly. Conclusion: Non-invasive positive pressure ventilation able to improve the recovery rate and quality of life of patients with acute exacerbation of COPD.
\end{abstract}

\author{
KEYWORDS \\ Non-invasive ventilation \\ COPD \\ Clinical nursing
}

\section{Introduction}

Chronic obstructive pulmonary disease (COPD) is a serious respiratory illness, the clinical features are airway obstruction, and incompletely reversible airflow limitation [1]. Studies had shown that combination of drugs with bi-level non-invasive positive airway pressure (BiPAP) require appropriate nursing management in the treatment of respiratory diseases, so that the incidence of complications is reduced and the therapeutic effect in patient is improved and consolidated $[2,3]$. BiPAP ventilator is an effective tool

Copyright ( 2015 Zongying Li

doi: $10.18686 /$ jn.v4i4.3

Received: September 16, 2015; Accepted: November 7, 2015; Published online: December 29, 2015

This is an open-access article distributed under the terms of the Creative Commons Attribution Unported License (http://creativecommons.org/ licenses/by-nc/4.0/), which permits unrestricted use, distribution, and reproduction in any medium, provided the original work is properly cited.

${ }^{*}$ Corresponding author: Affiliated Hospital of Xuzhou Medical College, Xuzhou, Jiangsu 221003, China. E-mail: lizongying2010@sina.cn for treatment of clinical respiratory failure, while it is rarely used in patients with acute exacerbation of COPD. In our hospital, BiPAP ventilator and the corresponding nursing were used for acute exacerbation of COPD patients, and the remarkable clinical results were achieved, and reported it as follow.

\section{Materials and methods 2.1. General information}

152 cases of acute exacerbation of COPD patients treated in our hospital from January 2012 to December 2014, who met the diagnostic criteria of chronic obstructive pulmonary disease, were selected [4]. Among the 152 cases, there were 100 males and 52 females, aged from $41-90$ years old, with the average age $(56.93 \pm 13.81)$ years old. Before treatment, all the 152 cases of patients were excluded from emergency intubation, coma, upper gastrointestinal bleeding, severe liver and renal dysfunction, psychiatric history and other symptoms, all of them had no contraindications with non-invasive positive pressure ventilation. After that, 
Table 1. $\mathrm{PaO}_{2}, \mathrm{PaO}_{2}, \mathrm{SPO}_{2}$, and $\mathrm{pH}$ values of different group.

\begin{tabular}{cccccccc}
\hline Group & Case & Age (years) & Gender (male) & $\begin{array}{c}\mathrm{PaO}_{2} \\
(\mathrm{mmHg})\end{array}$ & $\begin{array}{c}\mathrm{PaO}_{2} \\
(\mathrm{mmHg})\end{array}$ & $\begin{array}{c}\mathrm{SPO}_{2} \\
(\%)\end{array}$ & $\mathrm{pH}$ \\
\hline Experimental group & 76 & $58.12 \pm 13.77$ & 54 & $51.46 \pm 3.61$ & $66.03 \pm 6.08$ & $70.60 \pm 12.77$ & $7.18 \pm 0.40$ \\
Control group & 76 & $55.72 \pm 13.84$ & 46 & $51.71 \pm 3.51$ & $65.72 \pm 7.29$ & $72.82 \pm 10.54$ & $7.24 \pm 0.37$ \\
$x^{2} / t$ & & 1.073 & 1.871 & 0.688 & 0.285 & 1.170 & 0.962 \\
$p$ & 0.285 & 0.231 & 0.679 & 0.776 & 0.244 & 0.338 \\
\hline
\end{tabular}

152 cases were randomly divided into experimental group and control group. The general clinical data such as age, gender, $\mathrm{PaO}_{2}, \mathrm{PaCO}_{2}, \mathrm{pH}$, clinical manifestations and others of two groups were compared and the results showed no significant difference $(p>0.05)$, hence they were comparable. Details were shown in Table 1.

\subsection{Methods}

Patients in control group received conventional treatment, however besides the basis of the control group, experimental group received BiPAP ventilation and relevant nursing management. $3 \mathrm{~mL}$ of radial artery blood were collected from two groups of patients after 7 days of treatment and blood gas analysis were made by the United States OPTICCA Micro Automatic Blood Gas Analyzer and corresponding reagents. At the same time, the changes of indexes such as breathing, blood pressure, heart rate, brain natriuretic peptide, clinical effect, adverse reactions and other indexes were observed.

\subsubsection{Conventional drug treatment}

Patients underwent blood routine, ECG, blood gas analysis, chest X-ray and other related diagnostic examinations before treatment. Adjust patient position, control infection by antibiotics, oxygen supply, maintain acid-base as well as electrolyte level balance, and bronchodilators, anti-inflammatory glucocorticoid, and phlegm treatment administration, in order to stabilize the condition.

\subsubsection{Bi-level non-invasive positive pressure ventila- tion (BiPAP)}

BiPAP Vision ventilator which produced by US Respironics Company was selected, and mouth (nasal) mask was used for non-invasive positive pressure ventilation. Some features included, oxygen concentration can be precisely adjusted according to the patient condition, variety of respiratory data monitoring and respiratory curves can be clearly displayed, and function of powerful automatic leakage compensation system. Parameter settings as, S/T mode (pressure support ventilation/pressure control ventilation), respiratory rate of 8-25 times/min, IPAP was started with 5-6 $\mathrm{cmH}_{2} \mathrm{O}$, then increased to $12-24 \mathrm{cmH}_{2} \mathrm{O}$ slowly, expiratory pressure is slowly increased from 0 to $3-9 \mathrm{cmH}_{2} \mathrm{O}$ according to the patient's condition. Ventilation was made for 4-7 hours a day. The oxygen concentration is set at $30 \%-50 \%$. Adjust the oxygen flow rate according to the standard of $90 \%$ or more peripheral oxygen saturation, and ensure the tidal volume (VT) $>400-500 \mathrm{~mL}$. The inspiratory and expiratory pressure in addition to time of positive pressure ventilation was adjusted at all time according observed patient's symptoms [5].

\subsubsection{Ventilator-related management Psychological care}

Patients may don't understand or misunderstandings the usage and functions of ventilator, which would lead to doubt, worry, fear, resistance, and other adverse psychology. This will make the patient does not actively cooperate with treatment, and seriously affecting the treatment effect. Nurses should provide patients with ventilator-related knowledge, precautions, function, necessary psychological guidance and principle of ventilation, so that they can recognizes the importance of ventilator therapy. Recommend patient from try to wear masks, adjust position and tightness of mask, in the case of disconnecting with ventilator, for better adapt.

\section{Accessories preparation}

Appropriate nasal mask or face mask are selected according to the patient's face shape and size, in addition, the selected nasal mask or face mask should have regulator with adjustable tightness, in order to increase the comfort and adherence to treatment of patient. During treatment process, the air humidifying fluid required $250-300 \mathrm{~mL}$ of sterile distilled water, which would be poured into the humidifier before use each time. Also, we must prepare sterile distilled water and disinfectant as the humidification bottle needs to be disinfected while replacing [6].

\section{Parameter settings}

The relevant parameters should be set according to subjective feelings and treatment effect of patients. When adjusting the parameters, the characteristics of disease, changes of blood gas and other indexes of patients are monitored timely. This is in order to select the best parameter, improve the efficiency, therapeutic effect, and reduce discomfort of patients, so that they're able to recover quickly.

\section{Respiratory nursing}

During ventilator therapy, respiratory tract must be kept unobstructed all time, which is extremely important for the treatment. Nasal secretion of patients was cleared be- 
fore ventilation therapy, so that the respiratory tract of patients is clean and unobstructed. During treatment, longterm inhalation of humidified gas will limiting the cilia movement definitely, and plenty of various nasal or oral secretions may cause airway obstruction easily. Expectoration of sputum should be carry out during treatment, ask whether the patients breathe smoothly or have feeling of blockage in a timely manner, and remind patients to replenish.

\section{Daily care}

The diet of patients must be in accordance with the scientific guidance of clinical nutrition, because sufficient nutrition able to improve body function of patients and help them in better recovery. Treatment by using ventilator may cause indigestion, gastric distension and other adverse symptoms, we shall strengthen the daily application support and timely replenish calories, protein, etc. Patients must be provided a quiet, warm, confortable environment to rest, and a guidance of daily routine should be listed for patients to ensure getting enough sleep [7].

\subsection{Observation guides}

The blood gas indexes $\left(\mathrm{PaO}_{2}, \mathrm{PaCO}_{2}, \mathrm{pH}\right)$ and respiratory condition (based on Borg scale) were tested after 7 days of treatment. The clinical effect, adverse reactions, intubation numbers, number of patients died during hospitalization and hospitalization days were observed [8].

\subsection{Statistical methods}

In this paper, statistical analysis was processed on the relevant data of two groups by SPSS 17.0. Count data were analyzed by test and measurement data were expressed as $\bar{x} \pm s$ and analyzed by $t$ test, $p<0.05$ refers to statistically significant difference.

\section{Results}

3.1. Comparison of blood gas indexes and respira-

\section{tory status of two groups.}

From the comparison of blood gas indexes between Table 1 and Table 2, it was observed that all the indexes of blood gas analysis were significantly improved after 7 days of treatment $(p<0.05)$. Compared with control group, $\mathrm{PaO}_{2}$ and $\mathrm{pH}$ value of experimental group was increased significantly and their $\mathrm{PaCO}_{2}$ level was decreased significantly after non-invasive ventilation $(p<0.05)$. The results shows that the vital function of two groups were improved, however the treatment effect of experimental group was more pronounced. For the respiratory status (based on Borg scale), there was no difference between two groups, while two groups were significantly improved after 7 days of treatment but the experimental group shown better result than the control group $(p<0.05)$. See Table 2.

\subsection{Clinical effect}

The results showed that, there were statistically significant differences $(p<0.05)$ in the aspects of prognosis, endotracheal intubation and hospitalization time between two groups. As for the death index, although there was no significant difference between two groups, however, the mortality rate of experimental group was $6.58 \%$ lower than the control group, details was shown in Table 3. The clinical effect of the experimental group was significantly better than the control group, suggesting that the use of non-invasive ventilation improved the recovery rate of COPD patients and their quality of life.

\subsection{Adverse reactions}

During non-invasive ventilation, there were 6 cases of varying degrees of abdominal distention, 5 cases of facial indentation, 10 cases of dry mouth in the experimental group, all of them were relieved slowly with no special treatment.

\section{Discussion}

COPD is a common clinical disease, most commonly in

Table 2. Comparison of blood gas indexes and respiratory status of two groups.

\begin{tabular}{cccccc}
\hline \multirow{2}{*}{ Group } & \multicolumn{2}{c}{ Blood gas indexes after treatment } & \multicolumn{2}{c}{ Respiratory status (Borg scale) } \\
\cline { 2 - 6 } & $\mathrm{PaO}_{2}(\mathrm{mmHg})$ & $\mathrm{PaCO}_{2}(\mathrm{mmHg})$ & $\mathrm{pH}$ & Before treatment & After treatment \\
\hline Experimental group & $89.60 \pm 3.40$ & $44.88 \pm 4.35$ & $7.51 \pm 0.06$ & $7.65 \pm 2.10$ & $4.29 \pm 0.22$ \\
Control group & $79.84 \pm 5.31$ & $49.88 \pm 4.30$ & $7.41 \pm 0.29$ & $7.44 \pm 2.06$ & $5.15 \pm 0.41$ \\
$t$ & 13.179 & 6.749 & 13.049 & 0.617 & 14.985 \\
$p$ & 0.000 & 0.000 & 0.000 & 0.539 & 0.000 \\
\hline
\end{tabular}

Table 3. Comparison of clinical effect between two groups.

\begin{tabular}{ccccc}
\hline Group & Case & Recovery and discharge (case/\%) & Endotracheal intubation (case) & Death (case) \\
\hline Experimental group & 76 & $60 / 83.33 \%$ & $5 / 6.58 \%$ & $2 / 2.63 \%$ \\
Control group & 76 & $48 / 63.16 \%$ & $18 / 23.68 \%$ & $7 / 9.21 \%$ \\
$x^{2} / t$ & - & 4.606 & 8.658 & 2.953 \\
$p$ & - & 0.032 & 0.003 & 0.086 \\
\hline
\end{tabular}


the elderly population. Acute exacerbation of COPD patients often suffer from cough, vomica, dyspnea and other clinical symptoms. In general, they may also have heart failure, such as acute exacerbation of COPD combined with acute left heart failure, leading to more great difficulty in treatment $[9,10]$. Generally, bi-level non-invasive positive pressure ventilation (BiPAP) provides auxiliary positive pressure ventilation by nasal (facial) mask directly, and with the continuous improvement and development of ventilator technology, it has been widely used in clinical. Compared to conventional ventilator, the biggest advantage of non-invasive ventilation is avoiding tracheotomy, and further evading the incidence of pneumonia, lung infections, etc. In the treatment of respiratory disease, combination of drugs with bi-level noninvasive positive pressure ventilation (BiPAP) can significantly reduce the endotracheal intubation rate, shorten hospitalization time, improve patients' symptoms, and has a noticeable effect.

In the process of traditional management, targeted nursing is not given according to the actual situation of noninvasive ventilation and patient's symptoms, so that the oral cavity and respiratory tract of patients are prone to blockage or bacterial growth, leading to some difficulties in treatment, and in severe cases, it is also easy to cause adverse reaction. In this study, ventilation nursing which provided for patients with acute exacerbation of COPD based on the characteristics of non-invasive ventilation, showed enhanced recovery rate and quality of life of COPD patients.

Compared with the control group, $\mathrm{PaO}_{2}$ and $\mathrm{pH}$ values of experimental group were increased obviously and their $\mathrm{PaCO}_{2}$ was decreased significantly after non-invasive ventilation. The results showed that the bi-level non-invasive positive pressure ventilation (BiPAP) can significantly improve blood gas, and help the rehabilitation of patients. As for respiratory status (based on Borg scale), the improvement of symptoms of experimental group was better than the control group; in addition, experimental group was better than the control group in the aspects of prognosis, endotracheal intubation, hospitalization time, etc. As for the death index, although there was no significant difference between two groups, the mortality rate of experimental group was $6.58 \%$ lower than the control group, suggesting a better clinical effect in experimental group. Although there are some adverse side-effect in use of BiPAP, but they don't needed special treatment and has minor effect on patients.

\section{References}

1. Wang $Q$. Nursing of noninvasive ventilation in treatment of chronic obstructive pulmonary disease combined with respiratory failure. Chinese Community Doctors, Medical Professionals. 2011;13(14):248.

2. Gou QJ. Nursing of non-invasive ventilation in treatment of chronic obstructive pulmonary disease combined with respiratory failure. Chinese General Nursing. 2011;9(11):2929-2930.

3. He F. Application value of BiPAP ventilator in acute exacerbation of COPD combined with acute left heart failure. Guide of China Medicine. 2012;12(10):184-185.

4. Wang $\mathrm{N}$, Lin $\mathrm{YH}$. Clinical observation of noninvasive positive pressure ventilation for acute exacerbation of COPD combined with type 2 respiratory failure. Chinese Journal of Difficult and Complicated Cases. 2012;11(11):846-848.

5. Gu XQ, Huang DZ. Effect evaluation of non-invasive ventilation on treatment of elderly acute exacerbation of chronic obstructive pulmonary disease combined with respiratory failure. Guide of China Medicine. 2012;30(10):22-23.

6. Mei J, Du J. Nursing of noninvasive ventilation in the treatment of chronic obstructive pulmonary disease combined with respiratory failure. Journal of Guiyang Medical College. 2011;36(05):542,544.

7. Su WL. Nursing of 42 cases of patients with chronic obstructive pulmonary disease combined with respiratory failure by non-invasive ventilation. Chongqing Medicine. 2010;39(11):1468-1469.

8. Wang T. Clinical nursing experience of non-invasive ventilation in treatment of COPD combined with type II respiratory failure. Medical Information. 2013;9(26):469.

9. Wang N, Lin YH. Clinical observation of noninvasive positive pressure ventilation for acute exacerbation of COPD combined with type 2 respiratory failure. The Northern Pharmaceutical. 2013;11(10):192-193.

10. Wang GH, Liu YM. Comparison of effect of non-invasive ventilation with or without independent piezometer tube on elderly type II acute exacerbation of chronic obstructive pulmonary disease combined with II type respiratory failure. Clinical Misdiagnosis \& Mistherapy. 2010;23(5):410. 